\title{
IdeAs
}

Idées d'Amériques

$11 \mid 2018$

Modernités dans les Amériques : des avant-gardes à aujourd'hui

\section{Bill \& Carlos : les Amériques de William Carlos Williams}

Bill \& Carlos: The Americas of William Carlos Williams

Bill \& Carlos: las Américas de William Carlos Williams

\section{Anna Aublet}

\section{OpenEdition}

\section{Journals}

\section{Édition électronique}

URL : https://journals.openedition.org/ideas/2333

DOI : $10.4000 /$ ideas.2333

ISSN : 1950-5701

\section{Éditeur}

Institut des Amériques

\section{Référence électronique}

Anna Aublet, «Bill \& Carlos : les Amériques de William Carlos Williams », IdeAs [En ligne], 11 | 2018, mis en ligne le 25 mai 2018, consulté le 20 octobre 2022. URL : http://journals.openedition.org/ideas/2333 ; DOl : https://doi.org/10.4000/ideas.2333

Ce document a été généré automatiquement le 20 octobre 2022.

\section{(i) $\odot$

Creative Commons - Attribution - Pas d'Utilisation Commerciale - Pas de Modification 4.0 International - CC BY-NC-ND 4.0

https://creativecommons.org/licenses/by-nc-nd/4.0/ 


\title{
Bill \& Carlos : les Amériques de William Carlos Williams
}

\author{
Bill \& Carlos: The Americas of William Carlos Williams \\ Bill \& Carlos: las Américas de William Carlos Williams
}

Anna Aublet

\section{Introduction}

1 Dans son épigraphe au recueil Al Que Quiere (1917), William Carlos Williams convoque l'œuvre de Rafael Arevalo Martinez, contemporain guatémaltèque. Le poète inaugure son volume par un extrait du conte intitulé El Hombre Que Parecia un Caballo (1915) qu'il avait déjà traduit avec l'aide de son père ${ }^{1}$ :

Había sido un arbusto desmedrado que prolonga sus filamentos hasta encontrar el humus necesario en una tierra nueva. Y cómo me nutría! Me nutría con la beatitud con que las hojas trémulas de clorofila se extienden al sol; con la beatitud con que una raíz encuentra un cadáver en descomposición; con la beatitud con que los convalecientes dan sus pasos vacilantes en las mañanas de primavera.

I had been an adventurous shrub which prolongs its filaments until it finds the necessary humus in new earth. And how I fed! I fed with the joy of remulous leaves of chlorafile that spread themselves to the sun; with the joy with which a root encounters a decomposing corpse; with the joy with which convalescents take their vacillating steps in the light-flooded mornings of spring. (Williams, Collected Poems: Vol 1, 481)

2 Le poète exhume déjà l'hybridité originelle de la terre américaine et de sa propre identité (Bill et Carlos) en faisant figurer sur la page les deux langues afin de mieux s'enraciner dans ce terrain réinventé : « the epigraph celebrates Williams' discovery of a poetic "new land" in which his bicultural, subterranean soul can flower » (Marzán, 127). Tout commence donc par la terre et ses gisements souterrains pour affleurer au ras du sol vers les feuilles d'herbe. Comme les filaments qui s'immiscent dans ce terreau fertile, le poète ravine, procède à une excavation du terrain pour accéder au sous-sol, à la géologie et à l'histoire de son continent et finalement atteindre le point de contact ${ }^{2}$ 
entre l'humus et les radicelles. Les racines explorent le sol à la manière du poème et de sa langue, elles sont « dynamiques, exploratrices, alimentent le sol aussi bien qu'elles le sondent, le nourrissent comme elles s'en nourrissent " (Darras, 345). On notera aussi que le poème inaugural de ce recueil, intitulé "Sub Terra", annonce déjà les expéditions archéologiques qui creusent un volume adressé "à celui qui veut » ou "celui qui aime", et déjà le docteur Williams nous entraine dans ce dédale translinguistique. L'épigraphe du recueil $\mathrm{Al}$ Que Quiere célèbre donc la nouveauté, les cryptes souterraines immaculées de l'Amérique et le sol poétique inventé par Williams, cet humus, rhizome vital pour la poésie moderniste américaine.

Al Que Quiere! - with its Spanish title, multilingual poem titles, and references to the multi-ethnic and immigrant communities Williams visited on his medical rounds - constantly alludes to the translocal and transnational roots of American culture encapsulated in his "Dedication for a Plot of Ground." These strategies reveal how Williams cultivated a rhizomatic conception of locality from which the poet's imagination radiated'. (White, 14)

3 C'est dans ce nouveau jardin que la double identité, l'hybridité du poète peut s'épanouir et devenir féconde. Pour Gabriela Hayden, à l'instar des premiers colons, le contact avec la nouveauté caractérise l'expérience latino-américaine : «Columbus's encounter with the "New World" of the Americas exemplified the encounter with newness that Williams came to believe was central to the (Latin) American experience " (Hayden, 176). Cet enracinement "volontaire et actif» (Marchessou, 12) sur le continent américain est primordial à la compréhension de l'œuvre du poète :

of mixed ancestry I felt from earliest childhood that America was the only home I could ever possibly call my own. I felt that it was expressly founded for me personally, and that it must be my first business in life to possess it. (Selected Letters, 185)

Citer Martinez revient également pour Williams à se frayer un chemin vers sa terre maternelle, l'Amérique Latine, Puerto Rico, sa matrie. La question de la réconciliation et de l'hybridation des identités poétiques travaille sans cesse la poésie williamsienne. Dans ses deux grands romans The Great American Novel (1923) et In the American Grain (1925), le poète se lance dans une quête des origines et de ses terres. Il est lui-même le produit de l'histoire erratique et mythologique du continent. Ces deux ouvrages préfigurent de façon macroscopique le long tracé cartographique à travers les chutes d'eau et les parcs boisés de Paterson, quelques années plus tard.

5 La quête initiatique et herméneutique de Carlos travaille l'œuvre du poète qui ne cesse d'interpeler Bill et de l'interroger: qu'est-ce que l'Amérique dans «littérature d'Amérique(s) » ? Le pèlerinage vers ses racines, à travers le continent, mène le poète à l'élaboration d'une histoire individuelle mais aussi collective : avec tout l'art chirurgical d'un médecin qui suture les chairs, le poète vient épaissir l'isthme du Panama, fine bande de terre articulant le corps des Amériques, et tisser son histoire personnelle sur la trame de l'histoire collective des Amériques. Lors de ses pérégrinations géographiques et poétiques, Williams effectue une sélection méticuleuse de ses modèles et de ses maîtres. Il crée du neuf à partir de l'ancien, redéfinit les contours d'un continent déjà érodé par l'histoire en faisant appel à l'âge d'or espagnol. Finalement, en liant ensemble l'ancien et le nouveau, l'américain et le portoricain, l'histoire des classes les plus riches à celle des plus pauvres, Williams se fait le tisserand d'une poésie américaine résolument métissée et moderne. 


\section{La cosmogénèse williamsienne}

\section{Etranger et barbare}

6 Dans son autobiographie, Williams évoque un poème non publié ${ }^{4}$, composé alors qu'il était encore étudiant en médecine à l'université de Pennsylvanie.

Like Endymion, it was a narrative in that vague area of thought associated with a romantic past. [...] It opened with a Keatsian sonnet for prologue, followed by an «Induction » that recounted in blank verse a tragic story ${ }^{5}$. (Autobiography, 59)

Le poète se remémore ensuite l'histoire d'un prince, seul survivant d'événements terribles qui décimèrent sa famille entière. Le récit débute in medias res, au réveil du prince dans un lieu qui lui est parfaitement étranger : "a foreign country» (ibid). Comme un écho à Rip Van Winkle dans la nouvelle de Washington Irving, le prince de Williams s'endort et ne reconnaît plus rien à son réveil. Le nostos, retour d'Ulysse vers son Ithaque natal, semble impossible. C'est donc un sentiment freudien d'inquiétante étrangeté (Unheimlich) qui s'empare du héro. Le prince se lance dans une quête épique pour retrouver sa maison (home), sa patrie.

This was no more or no less than the aimless wandering, for the most part, of the young prince in his effort to get home again as well as to discover what had happened to him - he had not been able to recall the details, merely "sensed" them: that there had been a beautiful bride, a father, a mother; that a disastrous event of some sort had occurred of which he was the victim. So he went on, homeward or seeking a home that was his own, all through a "foreign" country whose language was barbarous) ${ }^{6}$. (Autobiography, 60, je souligne)

Dans ce texte, le jeune Williams posait déjà la question du statut de l'autochtone, de l'étranger et du barbare. Les guillemets qui encerclent l'adjectif «foreign» viennent remettre en question la position ontologique de l'étranger et du local. A priori, le retrait des guillemets n'altère en rien le sens de la phrase. Pourtant, ils viennent matériellement indiquer la subjectivité d'un tel statut mais aussi le cadastre, la frontière qui divise les territoires et produit ainsi de «l'étranger» et du «local». Williams relate, à travers les périples du prince aux allures de Quichotte, sa propre préhistoire, celle d'un poète né du côté étasunien de la frontière, d'une mère portoricaine et d'un père anglais. La traversée du prince est aussi celle de ses parents lors de leur migration depuis le sud vers le nord du continent, événement marquant de la genèse du poète.

L'emploi du terme barbarous en position attribut de language vient lui aussi bousculer les points de vue. Le barbare fait bien originellement référence au langage et désigne celui qui ne parle ni latin ni grec, deux langues dont Williams contestait l'usage dans la poésie de certains de ses contemporains (notamment T.S. Eliot). Mais le barbare dans le contexte de la bal(l)ade lyrique de Williams, c'est aussi celui qui ne parle ni anglais ni espagnol, mais pratique l'idiome américain : «We are Americans; not only a material future but an unimagined future lies before us. We are a great people in the making. We are a new race, speaking our own language. We do not speak English, remember that. We speak our own language ». (in Wagner, 1969 : 139) Le sanskrit barbara évoque le bégaiement, les balbutiements d'une langue qui se fait le reflet des soubresauts et accidents de l'histoire. C'est pourtant cette histoire chaotique qui fit naître ce poète purement américain qu'est Williams, aux États-Unis, dans le New Jersey. 
I had begun to think of writing In the American Grain, a study to try to find out for myself what the land of my more or less accidental birth might signify ? (Autobiography, 178, je souligne)

10 L'expression « accidental birth » vient faire écho au poème « Catastrophic Birth » (CPII, 55) publié en 1944 et qui offre une définition poétique de la naissance pour le poèteobstétricien. L'ouverture (terrestre et charnelle) s'opère par craquements chez Williams et le poème révèle par l'image de la naissance doublée de l'éruption volcanique, la violence sous-jacente aux poussées de l'histoire.

Fury and counter fury! The volcano!

Stand firm, unbending. The chemistry

shifts. The retort does not fracture.

The change reveals-change.

The revelation is compact-

compact of regathered fury

By violence lost, recaptured by violence

violence alone opens the shell of a nut. [...] (CPII, 55)

11 Les répétitions ( fury ", " violence ", « change ", " compact ») tendent déjà à craqueler l'écorce («shell») de la montagne et du ventre gravide et préparent à l'écoulement final. L'antanaclase de « compact ", adjectif puis nom, l'anadiplose de « violence » et les innombrables redoublements tout au long du poème participent d'une forme de psittacisme qui se déploie par mimétisme contagieux dans la langue contractée et balbutiante : «A change stutters / in the rocks » (CPII, 56). Dans le même temps, les enjambements mettent en scène les premières coulées de lave et les premiers épanchements qui se traduisent dans le poème par des coulées de vers. De la "fracture », que l'on ne peut ici dissocier du sexe féminin, exhale le sens de la vie nouvelle: "the new opens / new ways beyond all known ways » nous dit le poète peu après (CPII, 56). Le poème se donne à lire comme prélude et avertissement à la conflagration de la bibliothèque au livre III de Paterson, composé cinq ans plus tard. Tel le Phoenix, la poésie renaîtra de ses cendres, " peace is reborn above the cinders » mais la bibliothèque est un monument stérile qui confine les œuvres: "The Library is sanctuary to our fears » $(P, 97-98)$ et c'est en Amérique Latine qu'il faut aller puiser l'inspiration créatrice.

L'accident, c'est la contingence, la circonstance, et c'est de cela que Williams tente de se faire l'amanuensis. Sur cette terre "étrangère ", le poète lâchera son cri de faucon, barbare lui aussi, sur les toits de l'Amérique, à la manière d'un Whitman avant lui. La question de l'étranger est au cœur de l'œuvre de Williams. Dans un essai sur Lorca publié deux ans après son assassinat par le régime franquiste en 1936, le poète se remémore un voyage en Espagne et sa prise de conscience de son statut d'étranger parmi les habitants de la région.

And I remember one night in 1910 in Toledo listening in the same way before a cubicle opening onto one of the plazas where a few men were sitting drinking. I went in, a young man not very familiar with the language and an obvious stranger, but they became self-conscious so that I took my drink and left soon after. (Selected Essays, 221)

13 Cet homme gêné et qui gêne, conscient de son statut c'est aussi Carlos face à Bill dont les identités ne peuvent être réconciliées qu’à travers la poésie. Dans une lettre à son ancien camarade de classe, Ezra Pound affuble Williams du costume de «bâtard des Caraibes » et l'exclut implicitement du cercle des «vrais " écrivains américains. Cependant, Williams n'était pas non plus associé à la littérature dite « ethnique » ou 
portoricaine, comme pouvait l'être son contemporain Arturo Schomburg. Dans ce contexte, In the American Grain, édification du mythe américain par Williams, peut se lire comme une réponse à Pound. Le livre déconstruit alors même qu'il élabore et invente un passé à l'Amérique réfutant ainsi l'idée que Williams, en tant que Caribéen, ne pouvait aborder le sujet de l'histoire américaine et encore moins se prévaloir de ses archives. En inventant les siennes, Williams répond aux arguments de Pound: "You thank your bloomin gawd you've got enough Spanish blood to muddy up your mind, and prevent the current American ideation from going through it like a blighted colander ${ }^{8} »$ (Imaginations, 11).

\section{Le Zeitgeist des années 20 et l'antipuritanisme.}

14 Le recours à l'espagnol dans les titres tels que «El Hombre » ou Al Que Quiere ainsi que les fréquents retours vers ses racines hispanophones semblent être pour Williams une façon de combattre un puritanisme latent qu'il abhorrait par-dessus tout. Pour Hayden, Williams réaffirme l'importance de l'expérience latine pour la construction identitaire américaine des années vingt, dans un contexte hostile au catholicisme : «He does this in the context of widespread prejudice against both Spanish and Latin American literature in the United States $»^{9}$ (Hayden, 190). En effet l'Espagne était encore considérée dans la première moitié du $\mathrm{XX}^{\mathrm{e}}$ siècle par les protestants comme le pays des papistes et de l'inquisition. La langue espagnole était elle-même hybride, parlée par de nombreux groupes ethniques différents. L'emploi de l'espagnol fait donc de l'œuvre de Williams une production véritablement anti-puritaine. Dans les années vingt, le puritanisme était le mot qui qualifiait le mieux les maux du pays (prohibition, ignorance, extrême pudeur) et Williams voyait la nécessité de se tourner vers une culture nouvelle, un sang nouveau pour sortir des carcans puritains, la nécessité de définir de nouveaux contours pour le continent à partir de lignes anciennes (« New contours suggested by old words », [IAG, 1]).

It was an ill that showed in prudery about sex, in Prohibition, in fear of plain talk, in all the usual manifestations of fastidiousness and decorum that William Carlos Williams came to hate, but most conspicuously showed itself in incessant pressure for conformity ${ }^{10}$. (Whittemore, 196)

In the American Grain, essai anti-puritain, devient ainsi un ouvrage résolument moderniste, un ouvrage de son temps.

\section{Un nouveau langage démocratique pour un sol fécond}

L'une des grandes questions soulevées par Williams est aussi celle de l'Histoire. L'histoire de l'Angleterre n'est pas l'histoire de l'Amérique, et la langue de l'Angleterre ne saurait être la langue des Américains. Comment le poète peut-il entreprendre de créer une langue en adéquation avec son sol, son sous-sol, son histoire et sa préhistoire ? Williams préconise l'invention, la création à partir des détails infinitésimaux qui se déploient sous ses pieds, à même le sol. Le poète doit se lancer dans le relevé des éléments autochtones de sa géologie pour reconstruire le passé et il le fait en suivant ses modèles espagnols, Góngora et Quevedo d'abord, puis Lorca. 


\section{Le parler ordinaire et le hic et nunc}

17 Pour Williams, on ne peut accéder à l'universel qu'en scrutant le particulier, en le mettant à nu pour l'examiner. Il nous dit que l'artiste ne doit pas chercher à agir sur la société mais s'y intégrer pleinement. Le poète ne doit donc pas appartenir à un bord politique défini ou à une confession, mais sa présence doit au contraire permettre de réconcilier ce qui est divisé, ou divorcé, pour emprunter un terme williamsien. Dans son autobiographie, Williams se revendique de Francisco de Quevedo chez qui il perçoit toutes ces valeurs. Il dit par exemple de la Novella Peregrine, un roman qu'il essayait de traduire :

The scheme of it is the putting down of the facts about the corrupt court, but not openly, which Francisco de Quevedo couldn't afford. Instead we have a story told in terms of proverbs of the people, among the scenes and songs of the farm where he then was staying ${ }^{11}$. (Selected Essays, 350)

18 C'est l'art poétique de Williams qui se trouve résumé dans ces quelques lignes. Transcrire ces "chants de la ferme" où logeait le poète espagnol, c'est ce que l'Américain se propose de faire entre Rutherford et Paterson, dans le New Jersey. Williams était particulièrement attiré par la transcription du langage ordinaire dans toute sa verdeur et sa vivacité, comme une sismographie de la langue que tout le monde pourrait déchiffrer.

Lors d'un discours prononcé à Porto Rico en 1941, Williams mentionne l'union entre les États-Unis et l'Espagne, qu'il oppose ensuite au mariage aberrant et même incestueux avec l'Angleterre ${ }^{12}$.

$16^{\text {th }}$ and $17^{\text {th }}$ century Spain and Spaniards are nearer to us in the United States today than, perhaps, England ever was. It is a point worth at least taking under consideration. We in the United States are climactically as by latitude and weather much nearer Spain than England, as also in the volatility of our spirits, in racial mixture - much more like Gothic and Moorish Spain ${ }^{13}$. (Mariani, 447)

Ainsi selon le poète, les conditions climatiques, intellectuelles et ethniques semblent présager de l'union harmonieuse entre l'Espagne et les États-Unis. Il trouve aussi dans la littérature espagnole une qualité particulière, la capacité de créer une poésie de l'ici et maintenant, du local. Il décèle cette propriété chez Lorca et tente d'intégrer cet élément à sa propre poétique. Pour Williams, non seulement le climat espagnol est plus proche du sien mais la géographie de l'Espagne a contraint ses habitants à se concentrer sur le sol " sous leurs pieds » : «Spaniards have remained basically limited to the reality at their feet from which there was no escape » (Selected Essays, 223).

Williams lit par exemple El Libro de Bueno Amor de Juan Ruiz comme un cante jondo populaire, une voix poétique, démocratique et musicale, fruit d'une société espagnole bigarrée et diverse : «Williams ici nous parle indirectement, à la manière de Quevedo, de sa lutte pour une poésie émanant du creuset des cultures présentes en Amérique et de la possibilité d'une voix unique et harmonieuse naissant de cette diversité » (Aji, 83). Ainsi, d'après Hélène Aji, Williams tente d'importer ce mode de métissage poétique dans la langue américaine et l'épique Paterson, si éloigné qu'il puisse paraître de l'influence espagnole, en sera l'illustration la plus probante. Ces apports divers contribuent à importer une part d'étrangeté dans la langue même, étrangeté qui caractérise pour Williams l'idiome américain :

-in the very accent

multiplied 
of the local idiom

they knew - (YCAL, 53/1224)

L'oscillation entre dialogue et action, le souci de l'ancrage dans un ici et maintenant, la transcription du parler commun sont autant de caractéristiques de la poésie de Quevedo reprises par Williams dans Paterson. Quant à Góngora, Williams dit de lui qu'il est « la carte même de l'Espagne $»^{14}$ comme lui-même se fera à travers Paterson, la carte de l'Amérique.

Grâce à ses origines et à sa culture métissée, Williams pense pouvoir trouver un langage pour dire la diversité, et donc accessible à toutes les classes, outrepassant toutes les couches de la population. En tant que médecin, il était le candidat idéal pour la tâche qu'il s'était fixé car il fréquentait toutes les couches de la population. Une fois encore, le docteur vient combler la béance et faire entendre la voix de la diversité.

\section{Rapatriement vers l'Amérique}

Finalement, Williams admirait Góngora et Lorca pour leur remise en question des modèles européens et l'invention de leur propre modèle. Cette idée est très clairement exprimée dans The Great American Novel : "Oh to hell with Masters and the rest of them. To hell with everything I have myself ever written ${ }^{15}$ » (Imaginations, 176). À la fin de son essai sur Lorca, Williams poursuit sa réflexion non sequitur sur Walt Whitman et rapatrie ainsi son discours vers l'Amérique. Il explique devoir continuer l'œuvre de Whitman qui n'a pas eu le temps d'aller assez loin, un peu à la manière de Lorca après Góngora ${ }^{16}$. L'essai sur Lorca vient donc réconcilier les deux identités de Williams. Bill et Carlos se trouvent harmonieusement réunis et c'est ce mariage que Williams pense fertile et qu'il oppose à l'alliance anglo-américaine. Alors qu'il était en pleine composition de son essai sur Lorca, Williams écrit ceci à son ami Kenneth Burke en 1937 :

So the world degenerates and I'm doing an essay on the development and significance of Spanish poetry. What a subject! and how much more important to us than the blight of English literature under which our cocks have all but rotted away into each other's asshole ${ }^{17}$ (in Mariani, 409).

Le langage fleuri employé ici par Williams marque bien sa conviction que l'union entre les deux pays n'est pas fécond et se trouve même en état de décomposition avancé. Comme le "decomposing corpse" de son épigraphe à Al que Quiere, il faut que le cadavre trouve un terrain fertile pour qu'il y ait une possible reviviscence. Williams cherche donc le contact continental et la fertilité de l'union entre l'Amérique du Nord et l'Amérique du Sud au contraire de cette terre aride et gaste qu'est pour lui le sol angloaméricain (dont T.S. Eliot était à ses yeux l'émissaire). Dans cette optique, l'Amérique, une fois débarrassée de ces vieilles grammaires poussiéreuses et des scories de l'idéologie de la Destinée manifeste, reste un terrain propice au surgissement d'une langue américaine. Le poète archéologue tente donc (et c'est bien là son paradoxe) de mettre à nu les couches préhistoriques d'une histoire littéraire à venir. Contradiction que Paterson tente de subsumer dans son titre même: " "pater" et "son", principe géniteur et héritier, racine latine et racine germanique, sujet et objet. Le nom de Paterson résume ce que Williams tire des traditions qu'il a envisagées: la possibilité d'être à la fois l'héritier d'un passé littéraire et le créateur d'un futur » (Aji, 86). Il faut, dans la mesure du possible, s'en tenir à l'indigénéité américaine, comme Lorca s'en tenait à l'indigénéité espagnole : «Garcia Lorca was especially important to Williams 
because he represented the poet who had remained in contact with the people, holding to an indigenous Spanish culture as opposed to the European tradition $»^{18}$ (Mariani, 409).

La solution préconisée par Williams réside dans l'hybridité même qui le constitue, c'est là qu'il puise le potentiel pour la nouveauté et l'invention. Dans un essai intitulé «The Basis of Faith in Art », le poète explicite ce pont établi entre la langue et l'invention : «so I began to invent, or try to invent, of course I had the advantage of not speaking English » (Selected Essays, 177). Non seulement, il ne parle pas anglais parce qu'il parle l'espagnol mais surtout, il ne parle pas anglais parce qu'il parle l'américain. Dans la langue espagnole, Williams, l'obstétricien obsédé par la nouveauté trouve un potentiel de renouveau pour une littérature américaine détumescente. Il se met en quête d'une langue qui serait faite pour la terre américaine et l'homme américain. Par le choix de l'hybridité, le poème devient ainsi un véritable mestizaje, métissage de brins et filaments d'origines diverses.

\section{III. (Mé)tissage du mythe américain}

Dans In the American Grain, Williams fait l'éloge du Père Rasles, un missionnaire jésuite lexicographe. Il admirait tout particulièrement la réponse respectueuse et tolérante de Rasles à l'égard du langage des Amérindiens.

It is this to be moral ... TO MARRY, to touch-to give ... to create, to hybridize, to crosspollenize ... ${ }^{20}$. (In the American Grain, 121)

Le livre enjoint le lecteur d'accepter l'hybridité de toute chose et la matière même du continent à la fois duelle et diverse, majestueuse et terrible.

\section{Structure feuilletée}

À travers son roman, Williams tente d'articuler le chaos de l'histoire, de dire la confusion. Écrire le mythe américain implique une «interpénétration » (crosspollination) de discours. La poétisation du réel et de l'expérience est inhérente au mythe. Williams déconstruit alors même qu'il génère du mythe et c'est là une caractéristique primordiale du mythe. Le territoire américain, la carte, l'histoire américaine elle-même se font mythe dans la mesure où ils produisent de la fiction, de l'écrit. Le mythe compris comme une superposition de couches de fiction doit donc être lu de manière diachronique, comme un chevauchement ou ce que Levi-Strauss appelle une "structure feuilletée " $^{21}$ et qui n'est pas sans rappeler les feuilles d'herbe d'un autre chantre de l'Amérique: Walt Whitman. Williams apporte donc au mythe américain son propre feuillet, sa propre couche d'engrais avec In the American Grain. Comme les différentes strates qui constituent le sol américain mentionnées dans l'épigraphe à $\mathrm{Al}$ Que Quiere, le mythe se compose aussi de couches manuscrites et archéologiques.

En ce sens, In the American Grain se donne à lire comme la cosmogénèse de Williams, une histoire de l'avènement et des premiers balbutiements du continent. Pour Williams, l'histoire mythologique du continent est en constante métamorphose et ne saurait être fossilisée et conservée en quelques volumes de bibliothèque. Cette définition du mythe correspond à celle du «mythe synthétique » telle qu'exprimée par Vera Kutzinski dans Against the American Grain, pour designer un texte fondateur ondoyant et modulable : «a founding fable that evolves from the simultaneous decomposition and supplementation of classical mythologies of Old Worlds, meaning Africa and Europe, as they meet again in the New World ${ }^{22}$ » (Kutzinski, 110). Lorsque Williams pose les jalons de sa propre 
histoire mythologique de l'Amérique, il articule, de fait, celle des Amériques au pluriel. Une fois encore, le médecin est à pied d'œuvre pour remettre en place les articulations démises. C'est que l'acte du tisserand prime, celui qui vient tisser le texte sur le sol américain, celui qui entrelace l'histoire des États-Unis à celle de l'Amérique du Sud: "With In the American Grain, Williams developed a work that both modeled and argued critically for an understanding of the Americas as linked by a shared history ${ }^{23}$ " (Hayden, 177). Le produit d'un tel acte est un métissage de textes et de fragments qui émaillent l'œuvre du poète. Alors que Williams exhume ses archives pour mieux les recréer, le lecteur est amené à voir que seul un tressage des filaments qui constituent l'Amérique pourra donner naissance à une langue américaine autochtone.

\section{Structure métissée}

The Great American Novel est pour Williams l'occasion de livrer une anecdote qui illustre bien son propre art poétique métis.

The word bayeta is merely Spanish for baize. Great quantities of this were made in England for the Spanish and Mexican trade, the major part of which was of a brilliant red color. In this way English baize became Spanish bayeta to the Indians of the American Southwest. Familiar with the art of weaving, these Indians unraveled the bayeta, retwisted it into one, two or three strands, and then rewove it into their blankets, which are now almost priceless. This old blanket was picked up by the author in a New Mexican corral, for the purpose of wiping his buggy axle. It was covered with filth and mud. A number of washings revealed this glorious specimen of the weaver's art ${ }^{24}$. (The Great American Novel, 206)

Cette petite feutrine, bayeta, est le fruit du travail du tisserand qui vient lui même découdre l'objet importé pour l'intégrer dans ses propres artefacts. Les filaments de l'épigraphe à $\mathrm{Al}$ Que Quiere deviennent ici les fibres d'une nouvelle matière poétique. Cette démarche artistique est également celle du poète dans son texte qui vient tresser ensemble des brins de cultures et de langues variés pour ne créer qu'un seul artefact qui sera vers ou prose. La réconciliation des identités de Williams est en marche dans le processus même de tissage du texte. Ce tressage est une façon d'absoudre le langage de son pêché originel, de son incapacité initiale à capturer et saisir.

Mais ce que Williams nous dit, c'est aussi que l'hybridité originelle et naturelle de la terre est enfouie et qu'elle doit être nettoyée avant d'être retrouvée, «a number of washings revealed this glorious specimen of the weaver's art " (voir supra). Cette forme de catharsis eschatologique est omniprésente dans son œuvre, elle passe même par la destruction de la bibliothèque au livre III de Paterson, symbole d'une littérature fossilisée : « Pour lui, la littérature canonique n'est rien de plus qu'une couche morte du palimpseste littéraire qui recouvre le sol et le rend infertile » (Aji, 88). Il s'agit pour le poète non d'un nettoyage qui consisterait à lisser les aspérités de la langue mais bien davantage d'une purge au sens le plus scientifique et méthodique du terme dans le but de retrouver l'authenticité de la langue américaine. Le sol de l'Amérique est recouvert de boue et de rouille et la tâche du poète est de creuser aussi profond que possible pour atteindre l'humus qui permettra l'invention et la création. En s'émancipant des maîtres européens et en particulier britanniques, Williams choisit une forme d'orphelinat lexical et s'exile vers l'Amérique du Sud et sa "matrie " pour ne pas sombrer dans le mutisme et façonner une nouvelle langue pour l'Amérique. 


\section{Coda: crypter et décrypter le sens}

33 Le poème "El Hombre » est un bon exemple de mise en pratique de ce métissage poétique et du travail de déchiffrage que doit effectuer le lecteur.

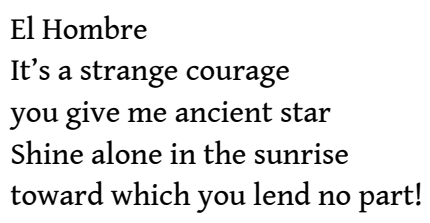

34 Le titre du poème semble déjà poser problème. Williams a expliqué dans un entretien qu'il avait choisi l'espagnol pour se démarquer de ses contemporains et éviter le français:

"El Hombre", because I was sick and tired of French titles. You know how the arty person loves to talk French?... Whether he can talk French or not, he always has to use a French term, that shows that he's smart, I suppose. Well, in order to change that around, I thought Spanish was a good language too, a very much neglected language since Longfellow and well,... Washington Irving! Yes. Irving and his "Alhambra » and Longfellow, they started off in Spanish ${ }^{25}$ ». (in Wagner, 1970 : 23-24)

La réponse sélective et désopilante de Williams ne dit pas tout de ce titre énigmatique car «El Hombre », c'est aussi Góngora: dans son essai sur Lorca, Williams s'exclame "Góngora was the man! ». Góngora serait donc aussi sans doute l'étoile ancienne ("ancient star ») dont l'éclat perdure dans la lumière de l'aube. Williams semble vouloir nous mener dans une quête herméneutique complexe. Il crypte le sens en oscillant dans un jeu étymologique de va-et-vient entre l'anglais et l'espagnol. Pour Julio Marzán, ce poème peut être interprété comme une excursion étymologique dans les langues, «a game of origins and etymologies» (Marzán, 3). Le sens se réserve, crypté dans le poème, et ne se s'offre qu'à la lumière des traductions successives. Pour Williams, la poésie saisit le sens de manière oblique, anamorphique : «Facts [whose] significance poetry catches aslant ${ }^{26}$ (YCAL, 54/1228). Le poème «El Hombre » est une belle illustration de cette anamorphose linguistique et oblige le lecteur à repenser le texte en changeant de point de vue herméneutique. Le terme « strange » réfère d'abord à ce qui est "étranger " et non "étrange » et se rapproche ainsi de "foreign ». De même, la racine latine de "courage " est bien "cœur » et l'étoile "star " peut être comprise comme une allusion à la mère de Williams, Elena, dont le nom dérive du grec signifiant lampe ou torche. Il est alors possible de lire la première strophe du poème comme suit : «It is a foreign heart / you give me old Helen ». Le poème creuse ainsi un sillon vers sa genèse, celle de sa mère portoricaine qui lui a donné ce double statut d'autochtone et d'étranger dont le poème s'efforce d'être le recueil.

Le poète n'emprunte que très peu de mots au lexique espagnol (à l'exception de quelques titres comme «El Hombre » ou Al Que Quiere par exemple) mais son œuvre est la trace d'une quête pour une langue américaine qui unirait les deux Amériques et elle s'imprègne de son héritage portoricain. Le nouveau mariage et l'hybridation qu'il préconise ( «an interpenetration / both ways » $[P, 4]$ ) ont lieu directement dans la langue poétique. Lorsqu'il revendique ses origines portoricaines, c'est avant tout un héritage dont Williams se réclame (Quevedo, Góngora, Lorca). Ces références se donnent à lire par le biais de rétro-traductions et de jeux étymologiques et non par des emprunts directs à l'espagnol. Semé d'archives et fragments de nature variée (lettres, 
journaux, livres d'histoire, publicités,...), le long poème Paterson illustre la volonté du poète de faire apparaître les coutures du texte, le poème nous est donné à lire «sur l'envers » et les fragments de toutes origines se hissent à la surface pour venir créer une nouvelle matière poétique composite qui fait l'originalité et l'authenticité de l'œuvre de Williams dans laquelle Bill et Carlos peuvent trouver leur place.

\section{BIBLIOGRAPHIE}

Aji, Hélène, Ezra Pound et William Carlos Williams: Pour une poétique américaine, Paris, l'Harmattan, 2001.

Darras, Jacques, « Le Grand Poème Américain », Revue française d'études américaines, 15 (1982): 343-371.

-_-_, Allen Ginsberg, la voix, le souffle, Paris, Jean Michel Place, 2002.

Dupeyron-Marchessou, Hélène, William Carlos Williams et le Renouveau du Lyrisme, Paris, Presses Universitaires de France, 1967.

Hayden, Gabriele, « New World Encounters: William Carlos Williams, Rafael Arévalo Martínez, and El Nuevo Mundo », William Carlos Williams Review 30.1 (2013): 181-199.

Kutzinski, Vera M., Against the American Grain: Myth and history in William Carlos Williams, Jay Wright, and Nicolás Guillén, Baltimore, John Hopkins UP, 1987.

Levi-Strauss, Claude, La Structure des Mythes, http://litgloss.buffalo.edu/levistrauss/text.shtml Mariani, Paul, William Carlos Williams: A New World Naked, San Antonio, Norton \& Company, 1990.

Marzán, Julio, The Spanish American Roots of William Carlos Williams, Austin, U. of Texas P., 1994.

Wagner, Linda, « William Carlos Williams: The Unity of His Art », Modern Language Association, Vol. 2, (1969), pp. 136-144.

-_-_, The Prose of William Carlos Williams, Middletown, Wesleyan University Press, 1970.

White, Eric B., "William Carlos Williams and the Local", The Cambridge Companion to William Carlos Williams, New York, Cambridge UP, 2016, p. 8-23.

Whittemore, Reed, William Carlos Williams, Poet from Jersey, Boston, Houghton Mifflin Company, 1975.

Williams, William Carlos, The Autobiography of William Carlos Williams, New York, New Directions Publishing, 1951 [Autobiography dans le texte]

-_-—, Selected Essays, New York, Random House, 1954.

-_-_, In the American Grain, New York, New Directions Publishing, 1956. [IAG dans le texte] Selected Letters. New York, Mc Dowell, 1957. 
-_-_, Imaginations: Kora in Hell / Spring and All / The Descent of Winter / The Great American Novel / A Novelette \& Other Prose, New York, New Directions Publishing, 1971. [The Great American Novel sous l'abbréviation GAN dans le texte]

, The Collected Poems of William Carlos Williams, Vol I. 1909-1939, New York, New

Directions Publishing, 1986.

———-, Paterson (Revised Edition), New York, New Directions Publishing, 1995.

, Archives de SUNY Buffalo (SUNYBUF)

\section{NOTES}

1. "My father, who really did the work in the translation of this story was accomplished in two languages, Spanish and English [...] Born in England he was brought to this country by his widowed mother at the age of five. When she married again, almost at once, he was taken to the West Indies where he grew up " (SUNYBUF, C97).

2. Terme cher à Williams puisque c'est ainsi qu'il intitula sa revue fondée en 1920 avec Robert McAlmon et parce que l'étymologie du mot concilie l'impression d'effleurement, de caresse charnelle et de toucher ferme et sans médiation du médecin qu'il était.

3. «Al Que Quiere! - avec son titre espagnol, ses titres de poèmes en plusieurs langues et ses références aux communautés métissées et issues de l'immigration que Williams côtoyaient lors de ses visites à domicile - ne cesse de faire allusion aux racines translocales et transnationales de la culture américaine, ce qu'illustre parfaitement "Dedication to a Plot of Ground". Ces stratégies sont révélatrices de la conception rhizomatique que Williams se faisait de la localité d'où l'imagination du poète irradiait » (ma traduction).

4. Trop imitatif de ses lectures romantiques de l'époque, le jeune poète insatisfait le jeta au feu.

5. «À l'instar d'Endymion, il s'agissait d'un récit ressemblant vaguement à l'idée que l'on se fait d'un passé romantique. [...] Il s'ouvrait sur un sonnet keatsien en guise de prologue, suivi d'une "initiation" qui relatait une histoire tragique en vers libres » (ma traduction).

6. «Il s'agissait ni plus ni moins, dans une large mesure, des errances d'un prince qui tentait de retrouver son chemin et de découvrir ce qui lui était arrivé - il ne lui restait aucun souvenir précis, juste des "sensations " : une magnifique mariée, un père, une mère et une sorte de tragédie dont il avait été victime. Il s'en était allé, en quête d'un chez lui, par une contrée "étrangère" à la langue barbare » (ma traduction).

7. « Je m'étais mis en tête d'écrire In the American Grain, un essai pour tenter de comprendre par moi-même ce que pouvait signifier la terre de ma naissance plus ou moins accidentelle.» (ma traduction)

8. «Grâce à ton sacredieu, ton sang espagnol t'épaissit suffisamment les idées pour que la grande idéation américaine en vogue ne puisse pas y passer comme à travers une purée de passoire » (ma traduction).

9. «Il met cela en œuvre dans une Amérique pétrie d'idées reçues contre les littératures espagnoles et latino-américaines » (ma traduction).

10. «C'était un mal qui se manifestait à travers la pruderie envers la sexualité, la Prohibition, la peur du parler franc, les manifestations habituelles de la bienséance et du décorum que William Carlos Williams était venu à détester, mais de manière plus évidente, ce mal se manifestait dans le poids suffocant du conformisme » (ma traduction).

11. «L'idée est d'exposer les faits concernant la corruption de la cour, mais pas ouvertement, car Francisco de Quevedo ne pouvait pas se le permettre. Au lieu de cela, il nous offre un récit fait de 
proverbes de tous les jours, auxquels il mêle les scènes et les chansons de la ferme où il séjournait » (ma traduction).

12. Voir infra.

13. «L'Espagne et le peuple espagnol des XVIe et XVIIe siècles sont plus proches de nous aujourd'hui aux États-Unis que l'Angleterre ne l'a sans doute jamais été. C'est un argument qu'il faut au moins prendre en considération. Aux États-Unis, nos conditions climatiques, météorologiques, nos coordonnées géographiques nous rapprochent bien plus de l'Espagne que de l'Angleterre. De même, l'inconstance de nos esprits, le métissage racial tiennent davantage de l'Espagne gothique et mauresque » (ma traduction).

14. "The map of Spain itself" (SE, 225).

15. «Au diable les maîtres et les autres. Au diable tout ce que j'ai moi-même écrit » (ma traduction).

16. «Schématiquement, Góngora, c'est Whitman ; Lorca, c'est Williams » (Aji, 82).

17. « Donc le monde dégénère et j'écris un essai sur le développement et l'importance de la poésie espagnole. Quel sujet! Et combien plus important pour nous que le joug de littérature anglaise sous lequel nos bites ont quasiment pourri dans nos trous du cul mutuels » (ma traduction).

18. «Garcia Lorca était particulièrement important pour Williams car il représentait le poète resté en contact avec le peuple et attaché à une culture espagnole indigène et non aux traditions européennes » (ma traduction).

19. « Donc je commençai à inventer, ou essayer d'inventer. Bien sûr, j'avais l'avantage de ne pas parler l'anglais » (ma traduction).

20. «C'est cela être moral... MARIER, toucher, donner... créer, métisser, hybrider...» (ma traduction).

21. Voir Levi-Strauss, 1957 http://litgloss.buffalo.edu/levistrauss/text.shtml.

22. «Un récit fondateur qui naît de la décomposition et de complémentarité des mythologies classiques des Vieux Mondes anciens mondes, (l'Afrique et l'Europe) lorsqu'ils se retrouvent dans le Nouveau Monde » (ma traduction)

23. "Avec In the American Grain, Williams a conçu une œuvre qui propose à la fois un modèle et une argumentation critique pour comprendre les Amériques à travers leur histoire commune » (ma traduction).

24. « En espagnol, le mot bayeta désigne la feutrine. De grandes quantités étaient fabriquées en Angleterre pour le commerce espagnol et mexicain, la plupart dans une couleur rouge vif. C'est ainsi que la feutrine anglaise devint la feutrine espagnole pour les Indiens du Sud-Ouest américain. Experts dans l'art du tissage, ces Indiens détissaient la bayeta, la torsadaient en un, deux ou trois brins, puis la tissaient de nouveau pour en faire des plaids dont la valeur est quasiment inestimable à l'heure actuelle. L'une de ces couvertures fut récupérée par l'auteur dans un corral du Nouveau-Mexique pour nettoyer un essieu. Il était couvert de saleté et de boue, et plusieurs lessives furent nécessaires avant que ce spécimen de l'art du tissage se révèle dans toute sa splendeur » (ma traduction).

25. " "El Hombre”, car je n'en pouvais plus des titres en français. Vous savez à quel point ces gens qui se la jouent artistes aiment parler français ? Qu'ils parlent ou non cette langue, il faut toujours qu'ils emploient un terme français pour montrer qu'ils sont intelligents, j'imagine. En fait, pour faire bouger les choses, je me suis dit que l'espagnol pouvait faire l'affaire, car c'est une langue délaissée depuis Longfellow et bien-sûr... Washington Irving! C'est ça. Irving et son « Alhambra » et Longfellow, ils ont commencé en espagnol » (ma traduction).

26. « Des faits dont la poésie saisit l'importance de manière oblique » (ma traduction). 


\section{RÉSUMÉS}

Dans son Autobiographie (1951), Williams qualifie le langage du Nouveau Monde, qui est pourtant le sien, de « barbare » jouant ainsi sur l'acception première et l'étymologie du terme. Il remet en question le statut ontologique de l'étranger qu'il est et qu'il n'est pas. La quête d'une langue purement autochtone traverse l'œuvre du poète et passe d'abord par la reconquête poétique de l'histoire et des mythes qui composent le continent. Dans ses deux grands romans, The Great American Novel (1923) et In the American Grain (1925) le poète effectue un long pèlerinage vers la genèse des Amériques et ne cesse de lier le destin des États-Unis à celui de l'Amérique du Sud, celle de ses origines, de sa mère portoricaine. De plus, l'excursion mène aussi à un métissage linguistique : l'emploi de termes espagnols, s'il peut parfois sembler arbitraire, vient encourager une lecture active du poème. Cet encodage sémantique semble en apparence contraire à la volonté de démocratisation de la poésie qui tenait à cœur au poète. Pourtant, nous tenterons de montrer comment Carlos relance souvent le jeu de la quête herméneutique en questionnant Bill, l'Américain intégré. Ce sont ces deux identités, Bill, dont la patrie est son New Jersey natal et Carlos, dont la matrie reste à Porto Rico, que le poète tente poétiquement de réconcilier.

As the sailors of William Carlos Williams' Great American Novel (1923) lay eyes upon the coast of America, they exclaim: "Nuevo Mundo!" The epiphany comes from a sensory point of view, which will be Williams's approach to his continent and its history. In his Autobiography (1951), Williams depicts the language of this new land-his own-as "barbarous," thus playing on the original meaning of the term. He keeps questioning the ontological status of the stranger that he simultaneously is and is not. The everlasting quest for a poetry of the hic et nunc and a primeval autochthonous language leads to a new poetic appropriation of the mythological history of the continent. In his two novels, The Great American Novel (1923) and In the American Grain (1925), the poet travels across the continent back to the origins of the Americas as he keeps entwining the history of the United States with the past of Latin America, his mother's land. Besides, the journey also leads him to a linguistic entwining: the use of Spanish words and phrases seems to activate a hermeneutic quest. Though this semantic encryption may appear at first as an obstacle to Williams's claim for a democratic poetry, it will be interesting to see how Carlos sets the hermeneutic process into motion by questioning Bill. Only through poetry does the poet succeed in reconciling his two identities: Bill, the native from New Jersey and Carlos, whose motherland remains in Porto Rico.

Los marineros de The Great American Novel del poeta William Carlos Williams (1923) observan la costa de América exclamando "¡Nuevo Mundo!". La epifanía viene desde un punto de vista sensorial, que será el enfoque de Williams en la narración de la historia de su continente. En su Autobiografía (1951), Williams describirá el lenguaje de esta nueva tierra extranjera (suya) como "bárbara", jugando así con el primer significado y la etimología del término. Pone en duda el estado ontológico del extranjero que ambos es y no es. La larga peregrinación por una poesía de un aquí y ahora y por un primigenio lenguaje autóctono lleva a una reapropiación poética de la historia mitológica del continente. En sus dos novelas, The Great American Novel (1923) y In the American Grain (1925), el poeta deambula por el continente hacia la génesis de las Américas y continúa vinculando el destino de los Estados Unidos con el de América del Sur, tierra de sus orígenes, su madre puertorriqueña. Además, la expedición también lo lleva a un entrelazamiento lingüístico: el uso de palabras y frases españolas viene a fomentar el desciframiento del significado. Esta codificación semántica parece en apariencia contraria al deseo de democratización de la poesía, querido para el poeta. Sin embargo, trataremos de mostrar cómo Carlos a menudo revive el juego de la búsqueda hermenéutica al cuestionar a Bill, el 
estadounidense integrado. Sólo es a través de la poesía que el poeta logra reconciliar sus dos identidades: Bill y su New Jersey nativo, y Carlos, cuya matria sigue siendo Puerto Rico.

\section{INDEX}

Mots-clés : William Carlos Williams, Modernisme, Hybridité, Nouveau Monde, Métissage, Mythe, Amériques

Keywords : William Carlos Williams, modernism, Hybridity, New World, myth, Americas, multiethnicity

Palabras claves : William Carlos Williams, modernismo norteamericano, hibridez, Nuevo Mundo, mito, Américas, mestizaje

\section{AUTEUR}

\section{ANNA AUBLET}

Anna Aublet est ATER à l'université Paris Nanterre où elle rédige une thèse sur les poètes William Carlos Williams et Allen Ginsberg sous la direction d'Hélène Aji. Elle a déjà publié sur les deux auteurs dans les revues Transatlantica, IdeAs ou encore Angles. Elle est lauréate des bourses de recherches de l'IDA et de l'ESSE. 